Article

\title{
Designing a Climate-Resilient Environmental Curriculum-A Transdisciplinary Challenge
}

\author{
Cheng-Yu Yu ${ }^{1, *}$ and Yi-Chang Chiang ${ }^{2}$ \\ 1 Department of Urban Planning and Development Management, Chinese Culture University, \\ Taipei 11114, Taiwan \\ 2 Department of Architecture and Urban Design, Chinese Culture University, Taipei 11114, Taiwan; \\ jyz4@ulive.pccu.edu.tw \\ * Correspondence: yzy9@ulive.pccu.edu.tw; Tel.: +866-2-2861-0511 (ext. 41105)
}

Received: 7 November 2017; Accepted: 22 December 2017; Published: 29 December 2017

\begin{abstract}
Building resilience is a promising transdisciplinary area that contributes to addressing the impacts of climate change. This paper focused on the design of climate-resilient environmental curriculums to enhance environmental quality. Transdisciplinary approaches have been recognized as being well-placed to assist responses to climate change, which is a complex phenomenon and problem. Semi-structured interviews were conducted to explore how climate-resilient environmental education can be integrated into the design, implementation, and practice transdisciplinary manner into curriculums. Transdisciplinary curriculum design is an important factor determining the quality of modules, especially in the field of environmental design, due to its real-life setting characteristics. As students are trained with new projects, and under different socioeconomic and environmental conditions, curriculum design requires modification. Moreover, promoting transdisciplinary studies is a new trend that influences curriculum design. Compared to the interdisciplinary approach, the transdisciplinary approach is concerned with issues and subjects that exist between disciplines, across different disciplines, and beyond all the disciplines, leading to an immense space of new knowledge. This approach leads to integrated research that involves non-academic participants. We concluded that the transdisciplinary approach is beneficial for students in two ways: better performance in practical modules, and addresses the real interests of the students. Feedback from students about the curriculum design suggested that, to consider the individual student's personal circumstances, multiple training methods should be used. The transdisciplinary approach to climate-resilient environmental curriculum design using a participative process amongst stakeholders is crucial; however, in this study, different opinions amongst interviewed tutors may obstruct the realization of the students' wishes.
\end{abstract}

Keywords: climate resilience; transdisciplinary approach; curriculum design; practical module

\section{Introduction}

Effective curriculum design is key to successful student learning and outcomes [1,2]. Environmental design involves the fields of architecture, landscape architecture, and urban planning and design, as well as the research pertaining to these fields. The bachelor in environmental design curriculum is practical-based education, which requires the completion of design-based studio experiences every semester, along with a number of complementary courses, general electives, and environmental design electives (http:/ / www.colorado.edu/envd/program-information/curriculum). Due to climate change and extreme weather, developing a transdisciplinary strategy that effectively integrates disciplines, approaches, and knowledge systems is necessary to create greater and more sustainable impacts $[3,4]$. This study was inspired by the fact that environmental design tends to be a practical discipline which, as Lang commented, " ... involves the use of a broad range of skills ... not only problem solving skills but 
also communication skills" [5]. Drawing upon Lang's recommendation, environmental design curriculum planning in Taiwan can be seen as a mixture of learning by doing and learning with doing [6,7], meaning studio practices and academic theories interact to enrich one another [8,9]. However, the academic modules offered by environmental design departments tend to focus only on the professional knowledge of the environmental design field, and do not include socioeconomic agendas and lack other presentational skills, such as communication skills and digital presentation skills. The heavy burden of compulsory modules limits the ability of students to study these agendas and skills. (For instance, students at the Department of Urban Planning and Development Management, Chinese Culture University had to complete 98 compulsory credits out of 132 graduation credits before 2015. Students of the Department of Architecture, Feng Chia University, had to complete 133 compulsory credits out of 156 graduation credits.)

However, some universities have a different curriculum. Harvard University and Massachusetts Institute of Technology have restructured the study program by allowing students to choose transdisciplinary modules in their first year of university, and declare their major in the second year. Transdisciplinary studies are believed to encourage students' motivation to study [10,11], allowing them to explore and determine their interests through first year studies and then decide upon a study area. This has influenced some proactive universities to adopt a similar approach to restructure their programs and modules, including the Chinese Culture University in Taiwan.

The Chinese Culture University has attempted to meet contemporary demands for a free environment to study from students and society as a whole. In 2015, the College of Environmental Design at the Chinese Culture University launched a scheme to reduce compulsory modules to allow students to choose modules according to their interests and abilities. The rationale for this action was not only to fulfill the university's policy, but to recognize that the complexity of climate-resilient issues could not be addressed by only one discipline [12,13]. Planning and design have traditionally concerned human welfare $[9,14]$, and have focused on physical planning concerning land use that has added other factors as society developed, such as social planning, housing, transportation, environmental planning, real estate development, universal design, and interior design $[15,16]$. After a series of social movements and changes in the late 20th century [17-19], planning and design have become comprehensive tasks, during which planners and designers must consider broader issues and the complex interaction between factors. Traditional planning and design theories and training, such as land use planning and landscape and architecture design, are essentially incapable of meeting society's demands for multidisciplinary planners and designers.

To address this situation, the College of Environmental Design implemented a policy that reduced the number of compulsory module credits to provide an opportunity for planning and design students to learn wider social, economic, environmental, technological, and psychological knowledge from other disciplines. According to the policy, three departments, Department of Urban Planning and Development Management, Department of Architecture and Urban Design, and Department of Landscape Architecture, considerably decreased the compulsory modules to allow students to choose their own modules. Moreover, intensive advertisement has encouraged the students in the College to choose modules in the other two departments within the College. To do so, the College, along with its three departments, established several transdisciplinary courses to guide students to take modules that complement specific knowledge and skills, such as planning and design theories and principles, computing simulation, contemporary societal debates, and legislation and regulations. The College of Environmental Design was the first college in Taiwan to integrate modules across planning, architecture, and landscape architecture departments. As a result, a small number of students in the three departments took the opportunity to choose modules from other disciplines. The students' motivation, experiences, and outcomes caught the attention of researchers. As a result, a series of research methods were used, and, consequently, this paper evaluated the extent to which transdisciplinary studies were beneficial to students, and provided feedback for curriculum design in the field of environmental design field. 


\section{Method}

\subsection{Context and Participants}

The practical curriculum researched in this study was in a newer college established in 1993, but was the first college in Taiwan to focus on environmental design, and two of its three departments were established in the 1960s. The practical aspect of the planning and design curriculum had been an important module for both teachers and students, and exists today in all three departments [11,20]. All three departments are similarly structured with about 60 students per class, and one class per grade. In the planning and design practical modules, students are divided into various groups based on the scale of sites, and each group has a tutor responsible for teaching and supervision. Each group completes an individual project. Students gather officially once or twice per week for up to $12 \mathrm{~h}$ in school, and regularly meet during off-school time to work on the projects. At the beginning of each semester, students are briefly introduced to the projects including clear requirements and assessment criteria. After the introduction, students conduct a site survey and investigation in following weeks, and organize and analyze the collected data. Each team subsequently provides the systematic survey results, including land-use planning and current conditions, a transport survey, public facilities, a hazard-prevention and emergency scheme, and open spaces distribution.

Although the three departments have different goals and focus on different planning and design scales, the approaches and techniques to planning and design are similar. All the information must be digitalized into computer software such as Autodesk AutoCAD and/or Geographic Information System software, i.e., ESRI ArcGIS, and presented in drawing maps. According to the analysis and understanding of the information, each team then gives a 15-18 min presentation of their vision and planning concepts for the designated areas, including the established physical development plans, and present their ideas using PowerPoint, two-dimensional (2D) and three-dimensional (3D) drawings, physical models, and a 3-min video clip. (Department of Urban Planning and Development Management requires each group of students to present a three-minute video clip from Years 1 to 4. Department of Landscape Architecture has a similar requirement, but only for Year 4 students. Department of Architecture and Urban Design has no such requirement). The presentation is assessed by a group including tutors and guests from public and private planning sectors, as illustrated in Figure 1. Assessors carefully review students' work and provide feedback, and the result is announced in the end of the presentation or shortly thereafter. The final rankings of each student are determined according to their performance throughout the semester and discussed by the panel of tutors to ensure the quality of rankings [21-23].

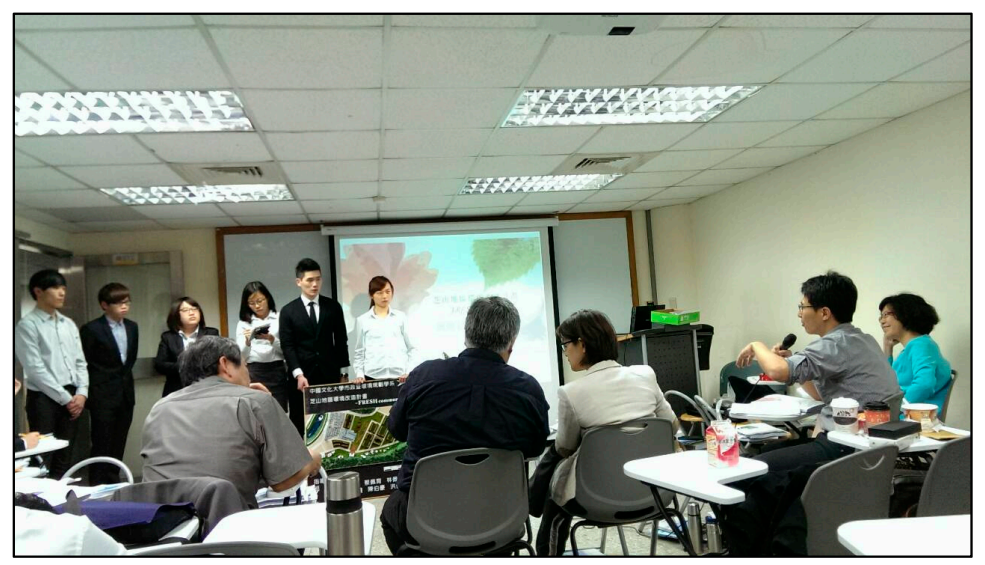

Figure 1. Final student presentation of their project, receiving the assessments and critiques of their tutors. A simulation of a typical assessment scenario; all students in the photo have graduated, and, in this case, assessors included guests: two architects, one governmental official, one manager from the private sector, and three tutors from the department. Some assessors are not in the scene. 
The curriculum design used to include a well-defined problem or set of problems within given areas for which students had to gather related information and use the natural or artificial advantages of the sites to solve the problems [21,24-26]. This curriculum design is a typical problem-oriented approach where students focused on understanding, defining, and solving problems [27]. During the curriculum restructure, the semester schedule of the curriculum was unchanged, but the curriculum design was altered. Instead of providing clearly defined problems for given areas, students were only provided a clearly defined area. Through their site visits and use of tools they had, such as sketch books, video recorder, cameras, and their five senses, students gathered the information required to define problems and analyze site conditions to create potential solutions. Students were questioned each week in terms of their observations, analyses, and solutions to provide guidance on the importance of knowledge of different disciplines. What students learned on site was beyond what had been taught in their departments, such planning and design theories and practices, and multiple-dimension issues, often intertwined, involving social, economic, and environmental considerations. As a result, students were encouraged to provide solutions in class rather than being criticized, considering their knowledge system had not yet been fully built. Asking for a complete solution for the defined problems would be unrealistic. This was a major change in the project design to encourage students to provide creative solutions rather than examining the workability of their solutions. That is to say, this change allowed students room for mistakes. The curriculum design was influenced by the solution-oriented approach defined by Kruger and Cross, but allowed solutions to be less practical. From this observation, the curriculum design was somewhat influenced by solution-based thinking that focused on methods to solve problems rather than tracing the roots of the problems $[28,29]$. The combination of thinking in the curriculum design, with learning by doing and learning with doing approaches, changed the traditional process of teaching and learning in Taiwan's planning and design program. Thus, the extent to which studying other disciplines could enrich and provide feedback to the planning and design knowledge and practices was therefore the center of this research, as shown in Figure 2.

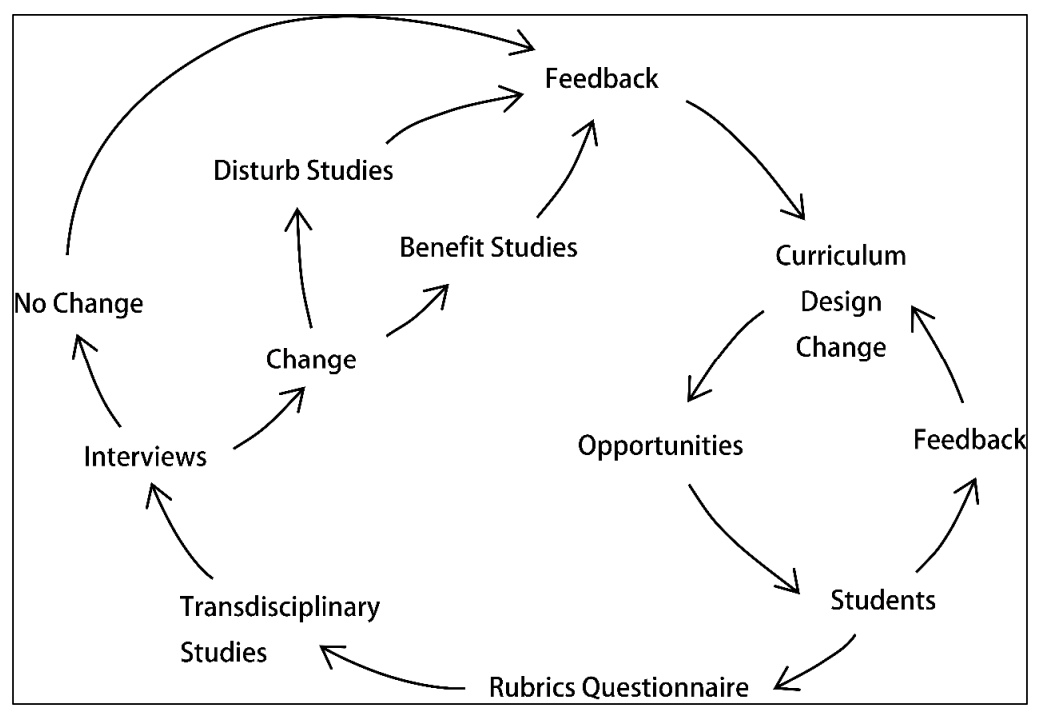

Figure 2. Research mindset.

\subsection{Data Collection and Analysis}

To understand the questions addressed in this research, we used multiple methods to generate data, and divided the data collection process into two steps. We began with a questionnaire survey followed by semi-structured interviews of tutors and selected students. The surveys and interviews were completed between April and June 2017 when the spring semester finished. As the researchers were the main implementers of the curriculum restructuring, they had the opportunity to observe the changes over two years. The field diary became another valuable source for the researchers to identify 
issues, track moments, and clarify ambiguities during the process of data analysis. Figure 3 presents the process of data gathering and analysis.

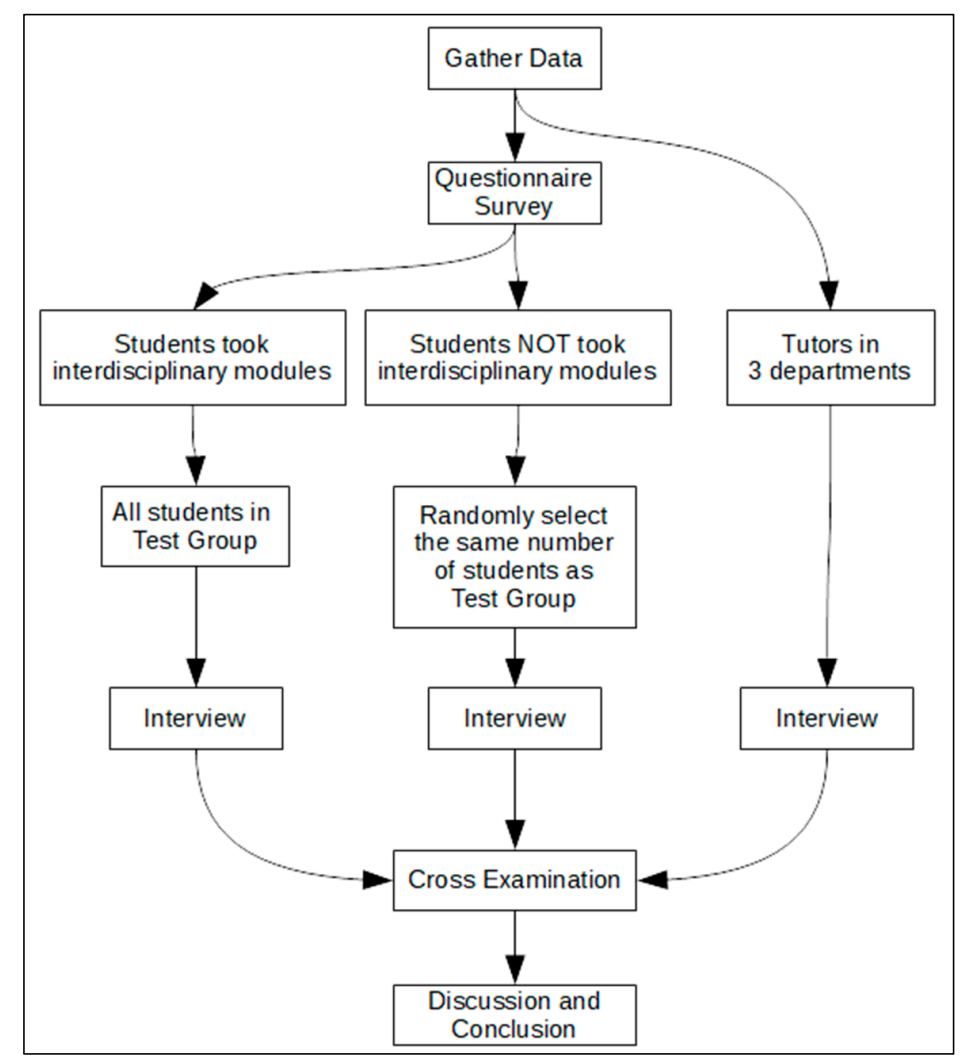

Figure 3. Research methods.

A questionnaire survey was used for students to evaluate themselves in terms of the changes in their gained or enhanced capacities and capabilities after the restructure of the curriculum, and the extent of the benefit they experienced by the dropped compulsory module credits. Before conducting the questionnaire survey, the researcher explained the purpose and use of the questionnaire survey to the students, and allowed opportunities to raise any concerns about the survey. In the questionnaire, students were asked to reflect on their status upon almost completing the annual studies at the university. After the briefing, an online questionnaire survey was sent to the students, and they were asked to complete it within two weeks. With permission, detailed personal information of the students was collected to enable researchers to identify specific students to conduct interviews later. In addition to the personal information questions, the survey included 31 questions, which were categorized into six sections: ability to present planning and design concepts, computer software skills, planning and design skills, confidence in themselves and professional areas, professional knowledge and skill building, and their experiences with transdisciplinary studies.

A typical five-level Likert Scale was used in the questionnaire, where 1 was strongly agree, 2 was agree, 3 was neither agree nor disagree, 4 was disagree, and 5 was strongly disagree. In total, 96 respondents completed this stage of the questionnaire.

For the second step, a semi-structured interview was conducted. Amongst the respondents, 22 students stated that they had chosen modules outside the department, as shown in Table 1, and they were interviewed. For a comparison, 22 students who did not take any module in other disciplines were randomly selected and interviewed as the control group. The questions asked covered: views on the approach they were taught to practical modules; the extent to which the approach was easy to understand, adopt, and apply; feedback on the practical modules to allow for improvements; and their rationale for not choosing transdisciplinary modules (control group only). 
The 22 students who chose transdisciplinary studies were asked additional questions about: their motivation to take transdisciplinary modules; their experiences in those modules; the extent to which these modules were beneficial to the practical module; and their feedback on curriculum changes.

Table 1. Number of students choosing modules in other academic departments.

\begin{tabular}{cccccc}
\hline & & Frequency & Percent & Valid Percentage & Cumulative Percentage \\
\hline \multirow{4}{*}{ Valid } & No & 74 & 77.1 & 77.1 & 77.1 \\
& Yes & 22 & 22.9 & 22.9 & 100.0 \\
& Total & 96 & 100.0 & 100.0 & \\
\hline
\end{tabular}

Students' annual transcripts were requested to review their achievements in the modules. The rationale for interviewing students was to confirm the survey results. In addition, the open-ended interview provided an opportunity to students to reflect on issues that were not known to the researchers [30,31]. In addition, six tutors (two tutors from each of the three departments) were interviewed after the student interviews were conducted, and the results were analyzed to understand different viewpoints on the issues raised in this research. Tutors were mainly asked about: their observation and personal views on the change in the practical curriculum design; students' response and achievements in the practical modules; other observations and thoughts they would like to share; and their views and responses to students' questioning and enquiries.

Quantitative and qualitative data were gathered and were analyzed using different techniques. Quantitative data analysis included bivariate and/or multivariate analysis to explore relationships between two or more variables. With the help of SPSS computing software, data analysis time was considerably reduced. Consequently, F-test, outputs of sum-of-square, and mean comparison were used via one-way analysis of variance (ANOVA). As a significant difference existed between variables, as shown through the F-test and sum-of-square comparison, the mean comparison was reviewed to investigate the difference. The chosen level of significance was $5 \%$. In addition, combined factors, such as grades combined with sex, were examined because the researchers anticipated that a difference would be found between the variables [32].

For qualitative data analysis, "Framework" was adopted. Framework is a data analysis method developed for conducting applied qualitative research using a unit base within the National Centre for Social Research in the U.K. (formerly the Social and Community Planning Research). "[It] involves a systematic process of sifting, charting and sorting material according to key issues and themes", and its strength is that " ... by following a well-defined procedure, it is possible to reconsider and rework ideas precisely because the analytical process has been documented and is therefore accessible" [33] (p. 117). The Framework procedure includes five stages: familiarization, identifying a thematic framework, indexing, charting, and mapping and interpretation. The researchers followed these five stages to analyze the qualitative data obtained from the interviews.

\subsection{Research Ethics and Insider-Outsider Issues}

This research was conducted in an ethical manner. As described, students who participated in the questionnaire survey were informed about the extent to which the results would be used and the students could freely choose to participate in the survey. Moreover, informed consent was provided to all interviewees, including both teachers and students, including questions may be asked during the interviews.

The researchers were aware of their positions in conducting this research within the college and departments, knowing that potential bias may occur from the students due to their relationships with and feelings toward the researchers. This insider-insider position [34,35] provides the advantage of gaining access to those being researched. However, this position may lead to the "value-action gap", in which the surveyed students provided answers they thought the researchers wanted [36]. Consequently, the researchers spent a considerable time to ensure the quality of data collected by constantly 
and repeatedly informing the interviewees about the purposes of this research and how the collected data would be used. In addition, students were informed that the survey would have no impact on their grades.

\section{Results and Discussion}

\subsection{Rubrics Briefing and Results}

Using the statistics results obtained with the SPSS software, Figure 4 presents the mean comparison between students who took transdisciplinary modules and those who did not. The former tended to believe they were more confident in communicative skills (Question 6 (Q6-Q9)) and data collection and analysis (Q14-Q16) compared to the latter. In terms of computing skills (Q10-Q13), two groups of students performed similarly without significant differences. An explanation for this finding is that 10 of the 22 students who took transdisciplinary modules chose computer software modules, especially in geographic information system and computer-aid drawing. The former group of students tended to agree that they were more interested in planning and design professions and would like to have a career in these fields after graduation (Q17-Q24).

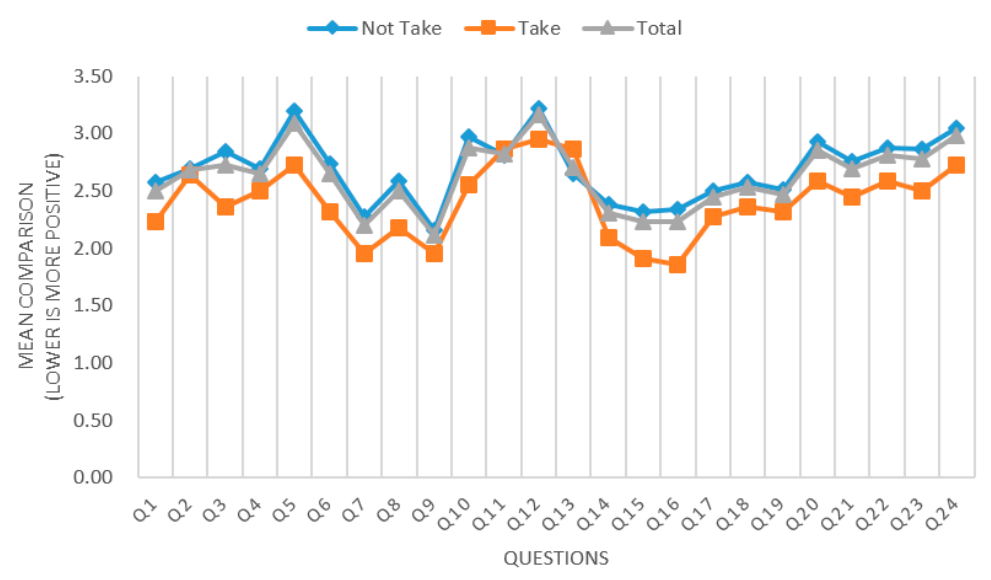

Figure 4. Descriptive group statistics using one-way analysis of variance (ANOVA) test comparing questions across variables.

Figure 5 presents the number of transdisciplinary modules that students chose in socioeconomic, natural science, computing, and other disciplines. These 22 students took a total of 75 transdisciplinary modules and were all in the top 10 in both their class annual transcript ranking and the practical module according to their transcripts and tutors' scoring records. Thus, these 22 students, along with 22 students who did not take transdisciplinary modules, were further interviewed and the results are presented in the next section.

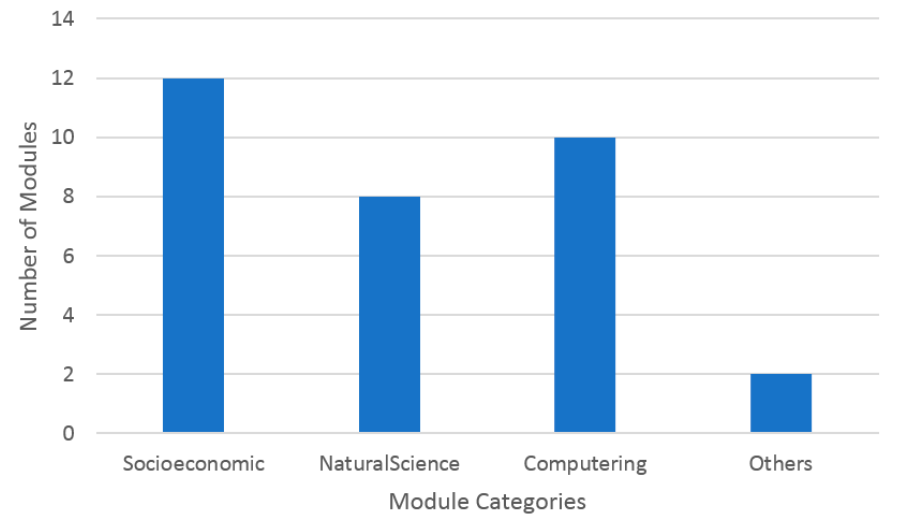

Figure 5. Numbers of modules taken in each category by students who took transdisciplinary modules. 


\subsection{Interview Results}

Interviews were conducted with 22 students each from the two groups, and two tutors from each of the three departments. The findings are presented in three sections: the comparison of the test group and control group, a detailed analysis of the students' responses in the test group, and cross-examination through tutors' responses.

\subsubsection{Comparison}

In general, students found the practical modules difficult and felt heavily burdened in all three departments, with similar responses from both the test and control groups. On average, according to their responses, the students had to spend at least $15 \mathrm{~h}$ per week to fulfill the weekly requirements from tutors. Although they were taught in the practical modules and professional knowledge was added from other modules in their departments, they emphasized that it was not enough to meet with requirements in the practical modules. This insufficiency was expressed in two dimensions: the speed of the practical modules' project schedules was too fast compared to the other professional modules, and the students felt that what they were taught was insufficient to complete the assigned tasks in the practical modules. This response raised a serious concern about the curriculum design, especially about the extent to which the pace of the practical modules should be in line with other professional modules, which is later discussed later along with the views of the tutors.

The learning by doing approach in the practical modules was acknowledged by the students, especially when tutors questioned less the practicality of their planning and design solutions and tasks. Instead, students were more confident in expressing their ideas and thoughts, with a focus on planning and design concepts and logical thinking, and skills they used to create the presentation. Moreover, the dialogue between students and tutors enriched the learning by doing approach that students believed is more useful than reading books and listening to taught programs in a classroom. Some students noticed that, as respondent S10 described below, even though the pace of practical modules was too fast compared to the other professional modules, as soon as the other modules covered the necessary material, they not only had a better understanding of the issues, but also the modules resonated with each other.

For example, we were asked to record traffic conditions in Year 1 and, at that time, we had no idea what this was about and whether the data were useful in practical modules. Tutors asked us to do the task, and we did it. When we took the Transportation module in Year 2, the most common feeling was: "Oh, that is it". We suddenly realized why we did traffic condition records in Year 1, and what and how the data could be used. (S10)

In addition to the pace of the practical modules, students recognized that what had been taught in the practical modules was easy to understand and adopt, but not all respondents agreed that it was easy to apply due to personal abilities, as respondent S28 described:

It is easier to know than to do. My studies have always been in the rational thinking route, which means I have no talent in art. Drawing and sketching require a sense of art, and I don't have it. I thought doing planning was all about writing reports, but why did we have to study drawing and sketching? (S28)

Consequently, students suggested that practical modules should involve multiple training methods according to students' personal abilities and interests so the practical modules would be more "attractive", increasing students' motivation to study. However, a small group of students made a radical suggestion. They claimed that not all students were interested in planning and design as multiple dimensions exist within the field. For example, students studying in the architecture department did not necessarily want to become architects. What they wanted to learn may just be general knowledge about architecture, and would potentially become knowledgeable real estate agents 
or construction material salesmen. In this situation, forcing them to spend considerable hours in planning and design drawings was unnecessarily, as respondent S35 described:

My wish was to become a successful real estate salesman. My rationale for studying in the architecture department was to understand architecture and enrich my knowledge. When I become a salesman, my clients will have more confidence in me. Since I did not want to be an architect, spending an extra $20 \mathrm{~h}$ a week or more creating architectural drawings was nonsense to me. (S35)

Various attitudes toward the practical modules were found amongst both groups of students who took and those who did not take transdisciplinary modules, as shown in Figure 6. According to the interview results, the rationale for not taking transdisciplinary modules was concentrated in three areas: the student did not yet know their life goals, the students already felt too much pressure in studying, and a lack of motivation for studying. The majority of respondents in this group maintained positive attitudes toward the practical modules. However, the results were interesting when they were compared to the other group.

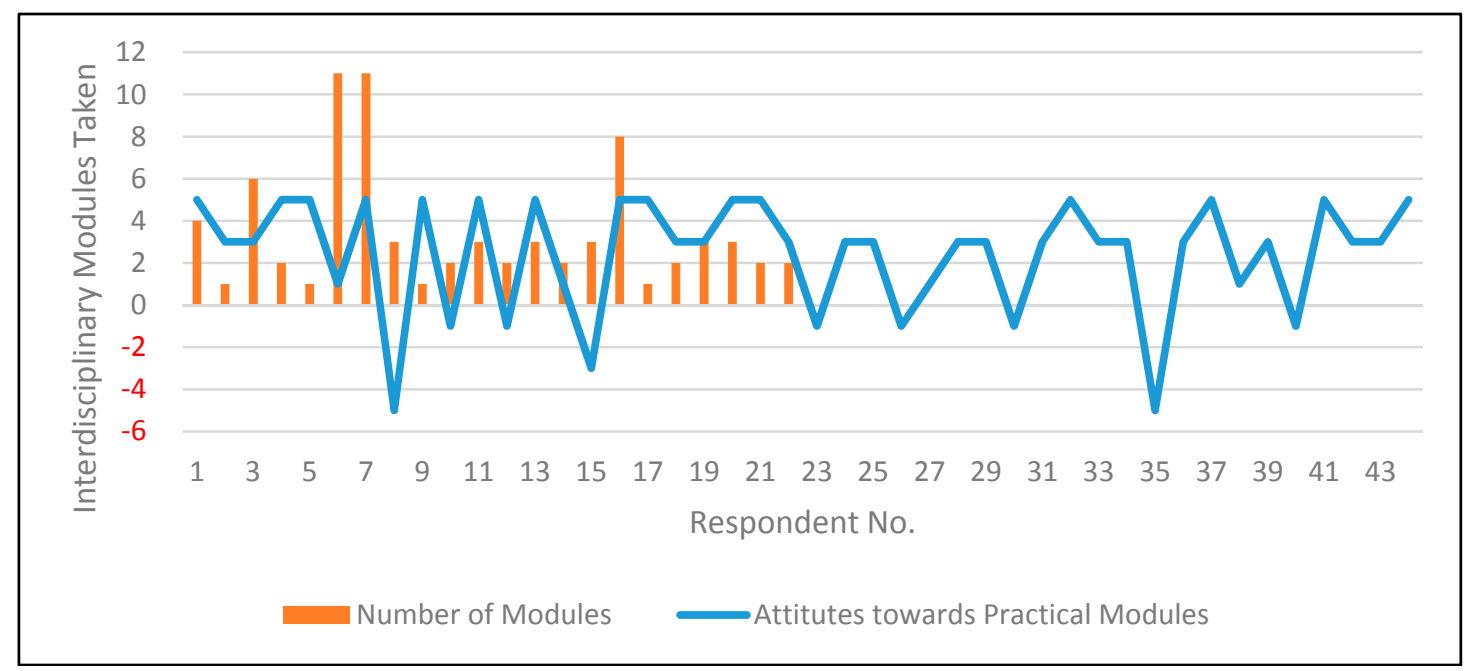

Figure 6. Four quadrant diagram: A comparison of attitudes toward practical modules between students who took (No. 1-22) and who did not take (No. 23-44) transdisciplinary modules.

\subsubsection{Transdisciplinary Studies}

Students who chose transdisciplinary studies did not necessarily have positive attitudes toward the practical modules, as shown in Figure 6. According to the respondents, three main reasons were the motivation to take transdisciplinary modules: recognizing the insufficiency of professional modules and knowledge taught in their department, enhancing their abilities in computer software, or disliking their department or practical module and wanting to study in a different professions or transfer to a different department. The first two reasons show the desire of students to improve themselves in the planning and design professions, and the last reason indicates that some students studied harder because they had found their real studying interests and began to prepare to transfer to a different department. Although they had different attitudes toward the practical modules, the students maintained relatively positive attitudes toward transdisciplinary studies because they had purposefully chosen those modules, as respondents S1 and S3 commented:

Two things made me take modules outside our department: one was that our department did not provide enough modules for us to choose, and the other was that some fields, such as economy and law, the specialists were not in our department. If I wanted to gain that knowledge, I had to take transdisciplinary modules. (S1) 
I wanted to improve my skills in 3D design and animated simulation because my senior colleague told me these were very important and helpful in my career, but our department only provided an introductory module without advanced modules. That was why I had to take advanced modules at the College of Journalism and Mass Communications and College of Arts. (S3)

Amongst these students, those who had positive attitudes toward the practical modules recognized the benefit from transdisciplinary studies for the practical modules. Those students who wanted to leave the planning and design professions also agreed that transdisciplinary studies benefited the practical modules, even though some ignored the modules, according to their annual transcripts and tutors' comments. In terms of their feedback on the curriculum changes, they tended to be positive about the changes, but, as previously mentioned, they also commented that individual abilities, capabilities, and interests should be considered when restructuring the curriculum. The inequality of talents, personal characteristics, locations of accommodation, group distribution, and even personal backgrounds and wealth were mentioned and emphasized, as some students had part-time jobs to sustain their livelihood and, as a result, were unable to spend as much time as others on the practical modules that encumbered the progress of their team. As a result, the students wished to participate in the curriculum design change process, allowing their voices and needs be heard, as respondent $\mathrm{S} 13$ commented:

\begin{abstract}
There was a decline in motivation to strive for the practical module amongst my classmates. The reasons were multi-dimensional. Some were fed up with the enormous tasks and challenges, some simply had no interest in the module whatsoever, some were busy doing their part-time jobs and could not work together with mates, etc. I do not think that tutors comprehend these conditions-tutors always said: "that is your problem, and you have to solve it". In fact, we are classmates and peer-pressure is weak nowadays, so we could either have someone in the team covers our work, or just let them pull the team back. Tutors have to seriously face the problem and restructure the curriculum in terms of task setup and group formation, and, most importantly, allow us to participate in the discussion. Therefore, our real needs would be understood. (S13)
\end{abstract}

Students' responses not only provided valuable and rich data for research questions, but also raised a series of concerns about the portions of curriculum design for which tutors were responsible. The feedback from the students was anonymously reported to the interviewed tutors, and their comments were provided at the end of interviews.

\title{
3.2.3. Tutors' Perspectives and Responses
}

The tutors generally considered the curriculum changes as routine. Most tutors told the researchers that the curriculum was constantly changing with every semester and every module, according to the changing environment and students. The difference with the practical module is that the changes were made collectively; all involved tutors worked together and decided how the module would be changed. For this task, tutors gathered issues generated from the latest semester and adjusted the curriculum for the next semester. Certainly, plans could never keep pace with the changes, and tutors often had meetings during semesters to modify the curriculum to adapt to immediate conditions.

We noticed that the bar for admission has continuously dropped, but students are increasingly proactive and creative in their interests. Consequently, tutors have been trying to adjust the curriculum design to include a diversity of content in the practical modules. The results and feedback were, however, not as positive as anticipated. Tutor E commented that:

Perhaps the tough training programs and enormous assignments scared many students in my class and made them stand back. As far as I know, there are at least six students who wanted to transfer to other departments or even universities. (Tutor E) 
To address this difficult situation, Tutor F suggested that the contents of the practical modules could be diversified, since students were more interested in computing and digitalized areas. Moreover, they pointed out that planning and design jobs are scarce in Taiwan, and tutors had to consider this when adjusting the curriculum, as they commented:

Recently, some students are interested in methods of presentation such as 3D models and animated simulations rather than seriously discussing on-site issues. Certainly, they will do well if they work in the graphic design and animation industry, and we have to acknowledge finding jobs in the planning and design field is difficult According to the last two years' survey, only $50 \%$ or fewer students work in the planning and design field after graduation. The job market simply cannot provide sufficient room for our students. Why don't we offer them more choices to develop their skills and increase their opportunities to have a job after university? (Tutor F)

On the other hand, Tutor B argued that planning and design skills could be separated into two areas: learning by repeated training and the organization and integration of multiple professions that may require talents, as they stated:

One important and basic skill in planning and design fields is drawing. Planners and designers rely heavily on drawing to communicate with others. Becoming Picasso requires a gift, but to have a sufficient skill in drawing only needs practice, practice, and practice. This is different from analyzing site data or producing great plans and designs. The drawing skill only requires time to build. (Tutor B)

Tutor A made a similar comment to emphasize the importance of training procedures and quality in the planning and design professions, as they warmed:

Planning and design follow a logical procedure, without such a procedure, students' ideas in planning and design might sometimes be unrealistic and cannot be implemented. The cause and effect ideology in the planning and design field is crucial to the creation of solution. Students need to be trained properly to gain the skills, especially concerning their jobs after university. (Tutor A)

Within the tutor group, arguments were made about what and how to teach students. Tutors had different approaches for the issues in e classrooms. Tutor D observed that some students adopted a proactive approach toward the challenges in the practical modules and their attitudes resulted in good achievements in the majority of the modules.

If students could survive the pressures in class, they generally performed well. Those students that either heeded our advice or proactively made decisions to choose modules in other departments performed better than others. The outcomes were significantly different than seen from their weekly homework, year review presentations, and final marks of modules, especially the practical modules. Their work was more mutual and holistic than others, when they applied knowledge from other fields to strengthen their views in particular. (Tutor D)

Therefore, judging which approach that is more suitable for students to incorporate into curriculum design is difficult. The fact is that each student is unique, and not all students can be accommodated by one set of teaching methods. Moreover, every year new students will have distinctive characteristics and they may not be able to adapt to previously successful curriculum designs. As a result, the curriculum design change cycle presented in Figure 2 should be conducted repeatedly for each year's students.

In terms of the questions and demands raised by the interviewed students, tutors had different views as well. Tutors all agreed that the pace of practical module was too fast and the pace of the 
related professional modules was inadequate. No proper method was in place to address the issue, as Tutor $C$ described:

If the pace of the practical modules is synchronized with the related professional modules, the pace of practical modules will be too slow. If the related modules want to catch up with the practical modules, students will not be able to sustain the pace, study the quantities of knowledge, and cope with the tasks and assignments in every module. Moreover, I wonder the extent to which students are capable of absorbing the knowledge, digesting it, and putting it into practice within such a short time period. Mission impossible. What we can do in the moment is to make the learning by doing approach easier for students to adopt than textbooks. (Tutor C)

Tutor C's description and Tutor F's comments addressed students' demand for multiple training methods according to students' personal abilities and interests. They agreed that students' innate and acquired inequalities should be respected and the curriculum should be adjusted in line with their personal circumstances. However, Tutors A and B disagreed with that viewpoint, as they emphasized the importance of proper training in planning and design professions. Tutor A further responded to this aspect and questioned students' circumstances:

Students' duty is to study. That is the meaning of going to university. If a student has to have a part-time job to sustain their livelihood, they should consider quitting university and finding a full-time job and, as soon as the expenses can be afforded, return to university to obtain the certificate. I have seen many students who could not do both things well and they learnt nothing or little from university. Some say that people with a senior high school diploma cannot find a proper job. I say that four years (typical university years) can conquer any difficulty if one works hard. To be honest, the purpose of having part-time job for many students is to buy computer, communication, and consumer electronics products like iPhones and pay for entertainment expenses, rather than sustaining them; it is just an excuse. If we push students harder, they will achieve more later. Believe me, they have potential and, do not forget, those who sow with tears will reap with songs of joy. We continuously tell students this principle every semester, but it seems that students do not remember it. (Tutor A)

This difference in opinion will continue endlessly as long as tutors' values are different. The same situation was found in response to the demand for participating in the process of curriculum changes. Tutors $\mathrm{C}$ and $\mathrm{F}$ believed that a participatory approach to curriculum design would be beneficial when they commented:

When departments hold module meetings, student representatives were invited to participate in the meetings to have their say. If we apply the same scheme to the curriculum design, students will have opportunities to express their views and demands and we can adjust the curriculum design accordingly. In the end, students will be willing to actively study the practical modules. (Tutor C)

Letting students have their say will help us to shorten the gap between teaching and learning, and, I believe, improve the teacher-student relationship. (Tutor F)

While Tutors $\mathrm{C}$ and F positively responded to the demand, Tutors A and B objected to the demand:

I cannot see the point of letting students participate in the curriculum design. If they have any comment, they can express their concerns in module meetings. There is already an official channel for them; we do not have to establish another one. (Tutor A)

We tried the participatory approach many years ago, but it failed. What happened was that student representatives had little to say because most students' opinions were not 
constructive. Many of their suggestions were unrealistic. That is why, I believe, it will be the same if we do it again. (Tutor B)

For this area, Tutor D suggested a compromise, calling for changes in tutors' attitudes toward students, as they suggested:

About 10 year ago we had similar scheme allowing students to express their concerns about curriculum design. Although we were implementing the university's policy, some achievements were made in my department. Nowadays students demand equality and justice in everything, we should accept their demands and see what happens. We can adjust the curriculum design anytime if something goes wrong. (Tutor D)

The differences in tutors' comments reflect the atmospheres in each department, as Tutor E commented:

Today's students tend to be self-centered and unless they know the reason for doing something, they would not follow the given orders. Students' attitudes toward the practical module between the three departments are considerably different. Students in other departments know their goals, but students in our department have little idea about what they will do and will become. As a consequence, even though the tutors in the three departments are tough and strict, their students managed to survive and conquer the difficulties. In contrast, our students have always tried to escape the pressure by collectively ignoring module requirements. In other words, tutors in the other two departments used to be dominating and had no sense of participation with students, whereas our tutors used to negotiate with students, and participation is not unusual in my department. (Tutor E)

Thus, the difference in views amongst tutors determined that whether tutors were willing to listen to students depending on the culture of the individual department. Nevertheless, tutors were aware of the issues raised by students, even though they addressed these issues differently according to their culture. In addition, the concept of participation was positive, but the participatory approach must be treated seriously and carefully, even if students are allowed to participate in the curriculum design process. Studies have shown that, due to its limitation and potential for manipulation, administrators and teachers have the power to decide who is invited to participate and what is to be discussed in the process $[37,38]$. Without a carefully designed scheme, the participatory approach to curriculum design could overwhelm students' voices [17].

\section{Conclusions}

Due to the impacts caused by climate change on the environment, a resilient strategy in the fields of urban planning, architecture, and landscape architecture design is urgently required. Consequently, academia has begun to adapt to the situation and realize the insufficient knowledge based on traditional planning and design theories and practices. We showed that the curriculum design changes in the practical modules in the College of Environmental Design at the Chinese Culture University have induced some students to take transdisciplinary modules to integrate different knowledge systems to address climate change issues. Although these students had different views and values toward the practical modules, their experiences in transdisciplinary studies were positive after completing modules in other departments, regardless of the taken number of modules. Moreover, the motivation to take modules in other departments stemmed from both positive and negative attitudes toward the practical modules. The positive students wanted to improve their abilities to successfully complete the projects in the practical modules; the negative students were realizing their real interests and preparing to leave their department by taking targeted department's modules. Both groups of students benefited from the experience. In addition, many students took advanced computer software modules to strengthen their technological abilities, which is becoming increasingly important when they pursue their careers $[39,40]$. 
The feedback from students about the curriculum was positive and practical, which included multiple training methods according to students' individual circumstances, and participating in the process of curriculum design changes. However, due to some tutors' conservative culture, these demands have not been treated seriously. In terms of curriculum design, studies and documents have addressed the way in which curriculum design occurs $[1,41,42]$. The difficulties in balancing the interests of teachers and students stems from teachers' cultures and the demands of the students [43]. The interactions between teachers and students also played an important role in teachers' willingness to change the curriculum as students may not pay enough attention to teachers [44] affecting the willingness of the teachers to offer change. As Tutor A claimed, if students want to learn, it is the obligation of teachers to teach, and the teachers are definitely willing to do so. Another appeal was that students wished to participate in the process of curriculum design to accomplish their interests. Due to a lack of consensus amongst teachers and the culture of the department, students' appeal has not been taken into account. However, teachers were aware of the pressures brought by students and there has been an official channel for student representatives to express views on curriculums in the disciplinary meetings of every department at Chinese Culture University.

To conclude, this paper has shown the challenges in curriculum design through an experimental study in the College of Environmental Design at the Chinese Culture University. Although changes in curriculum design, accompanied with other schemes such as cutting compulsory credits and promotion of transdisciplinary studies, encouraged students to take modules in other academic departments and seek out their real interests, difficulties occurred in the process of restructuring the curriculum due to a lack of mutual understanding between teachers and students. As the curriculum design is constantly being modified, as claimed by the interviewed tutors in this study, the researchers will continue to trace the changes in future.

Acknowledgments: This study has been supported by the staff and students at the College of Environmental Design, Chinese Culture University, especially the Dean and the Head of Department of Urban Planning and Development Management, Yang Song-Ling; the Head of Department of Architecture and Urban Design, Wen Kuo-Chung; and the Head of Department of Landscape Architecture, Monica Kuo.

Author Contributions: Cheng-Yu Yu was conducting the research project and writing the paper $(90 \%)$, supervised by Yi-Chang Chiang $(10 \%)$.

Conflicts of Interest: The authors have no conflicts of interest to declare.

\section{References}

1. Marzano, R.J.; Pickering, D.J.; Pollock, J.E. Classroom Instruction that Works; Association for Supervision \& Curriculum Development: Alexandria, VA, USA, 2001.

2. Dick, W.; Carey, L.; Carey, J.O. The Systematic Design of Instruction; Pearson Higher Education: San Francisco, CA, USA, 2005.

3. Bendito, A.; Barrios, E. Convergent Agency: Encouraging Transdisciplinary Approaches for Effective Climate Change Adaptation and Disaster Risk Reduction. Int. J. Disaster Risk Sci. 2016, 7, 430-435. [CrossRef]

4. Zscheischler, J.; Rogga, S.; Busse, M. The Adoption and Implementation of Transdisciplinary Research in the Field of Land-Use Science-A Comparative Case Study. Sustainability 2017, 9, 1926. [CrossRef]

5. Lang, J. Teaching planning to city planning students. An argument for the studio/workshop approach. J. Plan. Educ. Res. 1983, 2, 122-129.

6. Schank, R.C.; Berman, T.R.; Macpherson, K.A. Learning by Doing. In Instructional-Design Theories and Models: A New Paradigm of Instructional Theory; Lawrence Erlbaum Associates, Inc.: Mahwah, NJ, USA, 1999; pp. 161-181.

7. Felder, R.M.; Brent, R. Learning by doing. Chem. Eng. Educ. 2003, 37, 282-309.

8. Liu, L.-W. Local Connection and International Participation: A Teaching Experiment and Preliminary Practice on Real-World Problem-Solving, Intercollegiate Competition and Collaboration, and Transnational Interactive Learning. J. City Plan. 2007, 34, 363-383.

9. Innes, J.E. Planning theory's emerging paradigm: Communicative action and interactive practice. J. Plan. Educ. Res. 1995, 14, 183-189. [CrossRef] 
10. Apostel, L. Interdisciplinarity Problems of Teaching and Research in Universities; Organisation for Economic Co-Operation and Development: Paris, France, 1972.

11. Klein, J.T. Crossing Boundaries: Knowledge, Disciplinarities, and Interdisciplinarities; University of Virginia Press: Charlottesville, VA, USA, 1996.

12. Godemann, J. Knowledge integration: A key challenge for transdisciplinary cooperation. Environ. Educ. Res. 2008, 14, 625-641. [CrossRef]

13. Kramm, J.; Pichler, M.; Schaffartzik, A.; Zimmermann, M. Societal Relations to Nature in Times of Crisis—Social Ecology's Contributions to Interdisciplinary Sustainability Studies; Multidisciplinary Digital Publishing Institute: Basel, Switzerland, 2017.

14. Forester, J. Critical Theory, Public Policy, and Planning Practice; SUNY Press: Albany, NY, USA, 1993.

15. Chapin, F.S. Dr. Chapin Recalling the Early Days of the Department, i.e., The University of North Carolina Planning Department. 2010. Available online: http://www.theamericancity.org/wp-content/uploads / 2015/06/Urban-Land-Use-Planning.pdf (accessed on 28 December 2017).

16. Kaiser, E.J.; Godschalk, D.R.; Chapin, F.S. Urban Land Use Planning; University of Illinois Press: Urbana, IL, USA, 1995; Volume 4.

17. Yu, C.-Y. Public Engagement with Protected Area Policy: A Governmentality Perspective. Ph.D. Thesis, School of Geography and Planning, Cardiff University, Cardiff, UK, 2009.

18. World Commission on Environment and Development. Our Common Future; World Commission on Environment and Development: Oxford, UK, 1987.

19. Cullingworth, J.B.; Nadin, V. Town and Country Planning in the UK; Psychology Press: London, UK, 2002.

20. College of Environmental Design. History of the College. Available online: http://crt.pccu.edu.tw / files /111032-3039.php?Lang=zh-tw (accessed on 15 June 2017).

21. Yu, C.-Y.; Wang, S.-H.; Lee, D.-S. Module Introduction. Available online: http://icas.pccu.edu.tw/asst/ courses.aspx?dept=UTUPD\&fontsize=0.9\&iframe=courseInfo (accessed on 23 April 2017).

22. Lee, C.-L.; Kuo, W.-L.; Martinelli, A. Module Introduction. Available online: http:/ / www2.pccu.edu.tw / CRTDLA/data/\%E6\%99\%AF\%E8\%A7\%80\%E7\%B3\%BB(\%E6\%89\%80)\%E5\%AD\%B8\%E7\%BF\%92\%E5\% 9C\%B0\%E5\%9C\%96_980902.pdf (accessed on 26 January 2017).

23. Chiang, Y.-C.; Huang, K.-T. Module Introduction. Available online: http:/ / crtadp.pccu.edu.tw / files /141163-59679,r1221-1.php?Lang=zh-tw (accessed on 23 April 2017).

24. Urban and Rural Development Branch, Construction and Planning Agency. Manual of Urban Planning Procedure; Urban and Rural Development Branch, Construction and Planning Agency, Ministry of the Interior: Taipei, Taiwan, 2012.

25. Laseau, P. Graphic thinking for Architects and Designers; John Wiley \& Sons: New York, NY, USA, 2001.

26. White, E.T. Site Analysis: Diagramming Information for Architectural Design; ArchiBasX Press: Arlington, VA, USA, 2013.

27. Kruger, C.; Cross, N. Solution driven versus problem driven design: Strategies and outcomes. Des. Stud. 2006, 27, 527-548. [CrossRef]

28. Jonassen, D.H.; Hung, W. All Problems are Not Equal: Implications for Problem-Based Learning. Interdiscip. J. Probl. Based Learn. 2008, 2, 6-28. [CrossRef]

29. Mueller, S. Problem vs. Solution Focused Thinking. Available online: http:/ /www.planetofsuccess.com/ blog/2011/problem-vs-solution-focused-thinking/ (accessed on 16 April 2017).

30. Bryman, A. Social Research Methods; Oxford University Press: Oxford, UK, 2001.

31. Sarantakos, S. Social Research, 2nd ed.; MacMillan: Basingstoke, UK, 1998.

32. Bryman, A.; Cramer, D. Quantitative Data Analysis with SPSS Release 10 for Windows: A guide for Social Scientists; Routledge: London, UK, 2002.

33. Ritchie, J.; Spencer, L. Qualitative data analysis for applied policy research. In Analysing Qualitative Data; Bryman, A., Burgess, R.G., Eds.; Routledge: London, UK; New York, NY, USA, 1994.

34. Banks, J.A. The lives and values of researchers: Implications for educating citizens in a multicultural society. Educ. Res. 1998, 27, 4-17. [CrossRef]

35. Merriam, S.B.; Johnson-Bailey, J.; Lee, M.Y.; Kee, Y.; Ntseane, G.; Muhamad, M. Power and positionality: Negotiating insider/outsider status within and across cultures. Int. J. Lifelong Educ. 2001, 20, 405-416. [CrossRef] 
36. Blake, J. Community and Environmental Attitudes and Actions in Huntingdonshire: Report of the Baseline Research for the Huntingdonshire Going for Green Sustainable Communities Project; Committee for Interdisciplinary Environmental Studies: Cambridge, UK, 1997.

37. Hickey, S.; Mohan, G. Participation-From Tyranny to Transformation? Exploring New Approaches to Participation in Development; Zed Books: New York, NY, USA, 2004.

38. Ostrander, S.A. Democracy, civic participation, and the university: A comparative study of civic engagement on five campuses. Nonprofit Volunt. Sect. Q. 2004, 33, 74-93. [CrossRef]

39. Rose, D.H.; Meyer, A. Teaching Every Student in the Digital Age: Universal Design for Learning; ERIC: Alexandria, VA, USA, 2002.

40. Collins, A.; Halverson, R. Rethinking Education in the Age of Technology: The Digital Revolution and Schooling in America; Teachers College Press: New York, NY, USA, 2009.

41. Evans, N.J.; Forney, D.S.; Guido, F.M.; Patton, L.D.; Renn, K.A. Student Development in College: Theory, Research, and Practice; John Wiley \& Sons: New York, NY, USA, 2009.

42. Fishman, B.J.; Marx, R.W.; Best, S.; Tal, R.T. Linking teacher and student learning to improve professional development in systemic reform. Teach. Teach. Educ. 2003, 19, 643-658. [CrossRef]

43. Cronin-Jones, L.L. Science teacher beliefs and their influence on curriculum implementation: Two case studies. J. Res. Sci. Teach. 1991, 28, 235-250. [CrossRef]

44. Christophel, D.M. The relationships among teacher immediacy behaviors, student motivation, and learning. Commun. Educ. 1990, 39, 323-340. [CrossRef]

(C) 2017 by the authors. Licensee MDPI, Basel, Switzerland. This article is an open access article distributed under the terms and conditions of the Creative Commons Attribution (CC BY) license (http:/ / creativecommons.org/licenses/by/4.0/). 